\title{
Risk of synchronous endometrial disorders in women with endometrioid borderline tumors of the ovary
}

\author{
Shuang-zheng Jia ${ }^{1 \dagger}$, Jun-ji Zhang ${ }^{1 \dagger}$, Jun-jun Yang ${ }^{1}$, Yang Xiang ${ }^{1}$, Zhiyong Liang ${ }^{2}$ and Jin-hua Leng ${ }^{* *}$
}

\begin{abstract}
Background: Synchronous endometrial disorders have been poorly studied in women with endometrioid borderline ovarian tumors (EBOT). The aims of this study were to investigate the risk of endometrial disorders among women with EBOT and associated factors, as well as their oncological and fertility outcomes.

Results: This retrospective study included 33 women with EBOT. Their mean age was 41.9 years, and endometria were evaluated in 25 of these patients. The prevalence of synchronous endometrial disorders was $52.0 \%(n=13 / 25)$ and this incidence was $41.4 \%(n=46 / 111)$ after systematic analysis. Univariable analysis showed that EBOT patients who were younger, nulliparous, and had experienced abnormal vaginal bleeding were more likely to have synchronous endometrial disorders. The median follow-up was 54 months (range: 14-250 months), and three patients (10.3\%) developed recurrences. No deaths due to EBOT were recorded. Among the nine nulliparous women treated conservatively who were attempting to conceive, only one (11.1\%) pregnancy resulted in a live birth.
\end{abstract}

Conclusions: Synchronous endometrial disorders are common in women with EBOT, especially in those who are younger, nulliparous, and have experienced abnormal vaginal bleeding. Thus, endometrial sampling should be performed in women with EBOT undergoing conservative surgery, and a hysterectomy should be performed in cases requiring radical treatment.

Keywords: Endometrioid borderline ovarian tumor, Endometrial disorder, Fertility, Risk factor

\section{Background}

Borderline ovarian tumors (BOT), indolent neoplasms characterized by the presence of cellular proliferation and nuclear atypia in the absence of destructive stromal invasion, account for $10-20 \%$ of all epithelial ovarian cancers $[1,2]$. Over the past two decades, considerable work has been performed to investigate the clinicopathologic features of serous and mucinous BOTs, as well as the oncological and fertility outcomes of patients with BOTs [3-5]. However, due to its rare incidence, endometrioid BOTs (EBOTs), the third most common BOT, have been poorly studied [6]. Most studies on EBOTs have focused mainly on the pathological characteristics

\footnotetext{
*Correspondence: lengjenny@vip.sina.com

${ }^{\dagger}$ Equal contributors

'Department of Obstetrics and Gynecology, Peking Union Medical College Hospital, Chinese Academy of Medical Science \& Peking Union Medical College, Beijing 100730, People's Republic of China

Full list of author information is available at the end of the article
}

and included only brief descriptions of management and follow-up [6-10]. Additionally, only a few cases that were treated with conservative surgery have been reported; therefore, the fertility outcomes of these patients are unknown $[6,9]$.

Although approximately one-third of patients with EBOT have endometrial hyperplasia and/or carcinoma, to the best of our knowledge, no specific data are available that identify groups at high risk for EBOT [10]. Furthermore, staging surgery without endometrial biopsy remains the cornerstone for women with EBOT who hope to become pregnant. Thus, to better understand the course of EBOT and to improve the surveillance of these patients, a thorough exploration of the prevalence of synchronous endometrial disorders in women with EBOT and identification of potential risk factors is urgently needed.

The primary objective of our study was to investigate the risk of synchronous endometrial disorders among 
women with EBOT and to identify their risk factors. We also evaluated the oncological and fertility outcomes in these EBOT patients.

\section{Methods \\ Patients}

After obtaining approval from the Institutional Review Board at the Peking Union Medical College Hospital (approval No. S-K351), consecutive patients with EBOT who were treated or referred to our hospital were identified through a search of medical records between 1995 and 2015. Medical records, including patient charts and operative, pathological, and follow-up records, were comprehensively reviewed. All EBOT were diagnosed by two gynecologic pathologists at our institution, and the FIGO 2013 staging system for epithelial ovarian tumors was used for determining disease stage based on the operative descriptions and pathology records [11].

\section{Surgical management}

Conservative surgery was defined as fertility-sparing, wherein the uterus and at least part of one ovary are salvaged, whereas radical surgery was defined as bilateral salpingo-oophorectomy (BSO) with or without a hysterectomy. A proper staging surgery consisted of carefully inspecting all peritoneal surfaces with peritoneal washing, random or oriented multiple biopsies, and omentectomy [1]. For those patients referred to our hospital after EBOT diagnosis, a second surgery was offered. The extent of surgery was determined by the combination of previous surgeries and surgeries performed at our hospital. Pelvic lymphadenectomy and adjuvant platinumbased chemotherapy were performed according to the surgeon's preference.

Post-operative evaluation, including clinical examination, vaginal ultrasonography, and CA-125 measurements, was offered every 3 months during the first 2 years, every 6 months during the next 3 years, and yearly thereafter.

\section{Literature review}

We used MEDLINE and PubMed to perform a literature search. The following search terms were used: "borderline" OR "atypical" OR "low-malignant potential" OR "low malignant potential" AND "endometrioid" AND "tumor" AND "ovary" OR "ovarian". Only articles published in English were included.

\section{Statistical analysis}

Primary outcomes included coexisting endometrial disorders in women with EBOT, defined as endometrial cancer, atypical hyperplasia (AH), and endometrial hyperplasia without atypia, during primary surgery and/or follow-up. Data were expressed as mean \pm standard deviation, median (range), or number (percentage). For inferential analysis, an independent $t$ test and Fisher's exact test were used to compare study variables between cases (women with synchronous endometrial disorders) and controls (women with normal endometrium) when appropriate. Due to the small sample size, multivariable analyses were not performed. All data were analyzed using SPSS 23.0 and $p<0.05$ was considered significant.

\section{Results}

\section{Patient characteristics}

A total of 590 BOTs were identified, of which 33 (5.6\%) were diagnosed as EBOT. The mean age of the patients was 41.9 years (range: 23-81 years). More than twothirds of the participants (69.7\%) were premenopausal. Of the 16 nulliparous women, four were infertile prior to treatment of their ovarian tumor. Ovarian cysts were the most common indications for surgery $(75.8 \% ; 25 / 33)$ and elevated serum CA-125 values were found in more the half of the patients $(56.7 \% ; 17 / 30)$. The mean tumor diameter was $7.3 \mathrm{~cm}$ and bilateral tumors were present in 4 patients (including three recurring in the contralateral ovary). Demographic features and disease characteristics of the 33 patients are detailed in Table 1.

Approximately two-thirds of the patients were treated with laparotomy $(n=21)$, including five conversions from laparoscopy to laparotomy during a 2-step staging procedure. Among the 33 patients, 17 underwent conservative surgery and 16 underwent radical surgery after a one-step $(n=12)$ or a two-step procedure $(n=4)$. Staging surgery was performed for 18 patients, of which five were conservative. Fourteen patients underwent lymphadenectomy; no positive findings were recorded. Postoperatively, 10 women received chemotherapy and radiotherapy was prescribed to three women to treat synchronous endometrial cancer.

\section{Pathological findings}

Histological analysis revealed that 10 patients had coexisting peritoneal or ovarian endometriosis, including six that occurred in the ipsilateral ovary. Intraepithelial carcinoma (IEC) and stromal microinvasion were found in four and seven patients, respectively; all but two patients had stage I disease, including four patients with stage IC2. One patient with EBOT and microinvasion had an invasive implant of the sigmoid colon. Noninvasive implants of the omentum were seen in a 43-year-old woman with bilateral EBOT who had positive cytology as well as right ovarian IEC associated with EBOT.

\section{Synchronous endometrial disorders}

Twenty-five women had endometrial evaluations and 13 patients had synchronous endometrial disorders, including six women with endometrial cancer, five with 
Table 1 Demographic features and disease characteristics of participants

\begin{tabular}{|c|c|c|c|}
\hline Variable & n (\%) & Mean \pm SD & Median \\
\hline Age (y) & & $41.9 \pm 12.85$ & \\
\hline Nulliparous & $16(48.48 \%)$ & & \\
\hline $\begin{array}{l}\text { Preoperative serum } \\
\text { CA-125 }(n=30)\end{array}$ & & & $39.9(8-1037)$ \\
\hline$<35 \mathrm{IU} / \mathrm{L}$ & 13 (43.33\%) & & \\
\hline$>35 \mathrm{IU} / \mathrm{L}$ & 17 (56.67\%) & & \\
\hline Tumor diameter (cm) & & $7.3 \pm 4.56$ & \\
\hline \multicolumn{4}{|l|}{ Surgical extent } \\
\hline Conservative surgery & $17(51.52 \%)$ & & \\
\hline Radical & $16(48.48 \%)$ & & \\
\hline Staging surgery & $18(54.55 \%)$ & & \\
\hline \multicolumn{4}{|l|}{ Stage } \\
\hline । & $31^{\mathrm{b}}(93.94 \%)$ & & \\
\hline$\|-\| \|$ & $2(6.06 \%)$ & & \\
\hline \multicolumn{4}{|l|}{ Histological feathers } \\
\hline Stromal microinvasion & $7(21.21 \%)$ & & \\
\hline Intraepithelial carcinoma & $4(12.12 \%)$ & & \\
\hline Implants & $2^{c}(6.06 \%)$ & & \\
\hline Coexisting endometriosis & $10(30.30 \%)$ & & \\
\hline Endometrial evaluations & $25(75.76 \%)$ & & \\
\hline No lesions & $12(48.00 \%)$ & & \\
\hline Hyperplasia without atypia & $2(8.00 \%)$ & & \\
\hline EIN & $5(20.00 \%)$ & & \\
\hline Endometrial cancer & $6(24.00 \%)$ & & \\
\hline \multicolumn{4}{|l|}{ Additional treatment } \\
\hline Chemotherapy & $10^{d}(30.30 \%)$ & & \\
\hline Radiotherapy & 3 (9.09\%) & & \\
\hline
\end{tabular}

atypical hyperplasia $(\mathrm{AH})$, and two with endometrial hyperplasia without atypia. The crude prevalence of endometrial disorders in the women with EBOT was $52.0 \%(n=13 / 25)$.

Analyses of the data indicated that EBOT patients who were younger, nulliparous, and had abnormal vaginal bleeding (AVB) were more likely to have synchronous endometrial disorders $(P=0.02 ; P=0.03 ; P=0.001$; respectively; Table 2). However, no histological variables were found to be significantly associated with endometrial disorders.

\section{Oncological and fertility outcomes}

Four patients were lost to follow-up after surgery. Among the remaining 29 patients, the median follow-up was 54 months (range: 14-250 months). Three patients
Table 2 Risk factors of synchronous endometrial disorders in women with EBOT

\begin{tabular}{|c|c|c|c|}
\hline Variables & Cases $(n=13)$ & Controls $(n=12)$ & $p$ \\
\hline Age (y) & $39.1 \pm 10.4$ & $50.9 \pm 13.1$ & $0.02^{a}$ \\
\hline Menopausal & & & $0.11^{\mathrm{b}}$ \\
\hline No & 10 & 5 & \\
\hline Yes & 3 & 7 & \\
\hline AVB & & & $0.001^{b}$ \\
\hline No & 3 & 11 & \\
\hline Yes & 10 & 1 & \\
\hline Nulliparous & & & $0.03^{b}$ \\
\hline No & 5 & 10 & \\
\hline Yes & 8 & 2 & \\
\hline Tumor diameter & $5.7 \pm 2.1$ & $8.2 \pm 4.3$ & $0.07^{\mathrm{a}}$ \\
\hline CA-125 & & & $0.25^{\mathrm{b}}$ \\
\hline$<35 \mathrm{IU} / \mathrm{L}$ & 6 & 3 & \\
\hline$>35 \mathrm{IU} / \mathrm{L}$ & 5 & 7 & \\
\hline Coexisting endometriosis & & & $0.45^{\mathrm{b}}$ \\
\hline No & 10 & 8 & \\
\hline Yes & 3 & 4 & \\
\hline Stage & & & $0.28^{b}$ \\
\hline $\mathrm{H}-\mathrm{CC} 1$ & 11 & 8 & \\
\hline$|C 2-|||$ & 2 & 4 & \\
\hline IEC & & & $0.47^{b}$ \\
\hline No & 12 & 10 & \\
\hline Yes & 1 & 2 & \\
\hline Microinvasion & & & $0.46^{\mathrm{b}}$ \\
\hline No & 11 & 9 & \\
\hline Yes & 2 & 3 & \\
\hline
\end{tabular}

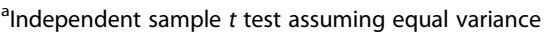

${ }^{\mathrm{b}}$ Fisher's Exact test

(10.3\%) developed recurrences but no women died of their disease. The time to recurrence among these 3 patients was 18 months, 100 months, and 180 months, respectively. These three women were young (23, 30, and 30 years old, respectively), nulliparous, and had coexisting endometrial disorders (two women with $\mathrm{AH}$ and one women with EC). Conservative surgeries without staging were performed at their initial treatment. Two women recurred on the residual contralateral ovary and one woman relapsed as bilateral recurrence (Table 3).

Among the 16 nulliparous women, 13 women were treated conservatively with a fertility-sparing procedure. One patient was lost to follow-up and three women had not attempted to conceive at the time of analysis. Of the nine patients who had attempted to conceive, only one $(11.1 \%)$ pregnancy resulted in a live birth. Moreover, of these nine women, five had synchronous endometrial disorders and two experienced contralateral EBOT recurrence. 
Table 3 Details of the three patients who experienced recurrence

\begin{tabular}{lllllll}
\hline Age & Initial surgery & Initial pathology & Time to recurrence & Secondary surgery & Secondary pathology & Coexisting endometrial disorders \\
\hline 30 & Lap UC+Hys & EBOT & 18 months & Lap BUC+D\&C & Bilateral EBOT & EC \\
30 & LSO+LN & EBOT & 240 months & Lap RSO & EBOT & EIN II-III \\
23 & RSO & EBOT & 100 months & Lap UC & EBOT & EIN II-III \\
\hline
\end{tabular}

Lap laparoscopy; Hys hysteroscopy; D\&C dilation \& curettage; UC unilateral cystectomy; EC endometrial cancer; LSO left salpingo-oophorectomy; RSO right salpingo-oophorectomy; EIN endometrial intraepithelial neoplasia

\section{Literature review}

We identified 347 citations from 1981 to November 2015. After reviewing titles and abstracts, only five papers that described 147 patients were included in the final analysis. Of these 147 women, 86 were evaluated for endometrial status. Thirty-three of these women were diagnosed with synchronous endometrial disorders, including nine who had endometrial cancer. Together with the present study, a total of 111 women with EBOT underwent endometria sampling and 46 were diagnosed with endometrial disorders, indicating a crude prevalence of $41.4 \%$ (Fig. 1).

\section{Discussion}

EBOT is a rare tumor that represents $0.2 \%$ of all epithelial ovarian tumors. To our knowledge, this is the first study to evaluate risk factors for synchronous endometrial disorders in women with EBOT. Our results indicated that synchronous endometrial disorders are highly prevalent in women with EBOT, especially in those who are younger, nulliparous, and have AVB. Our study also

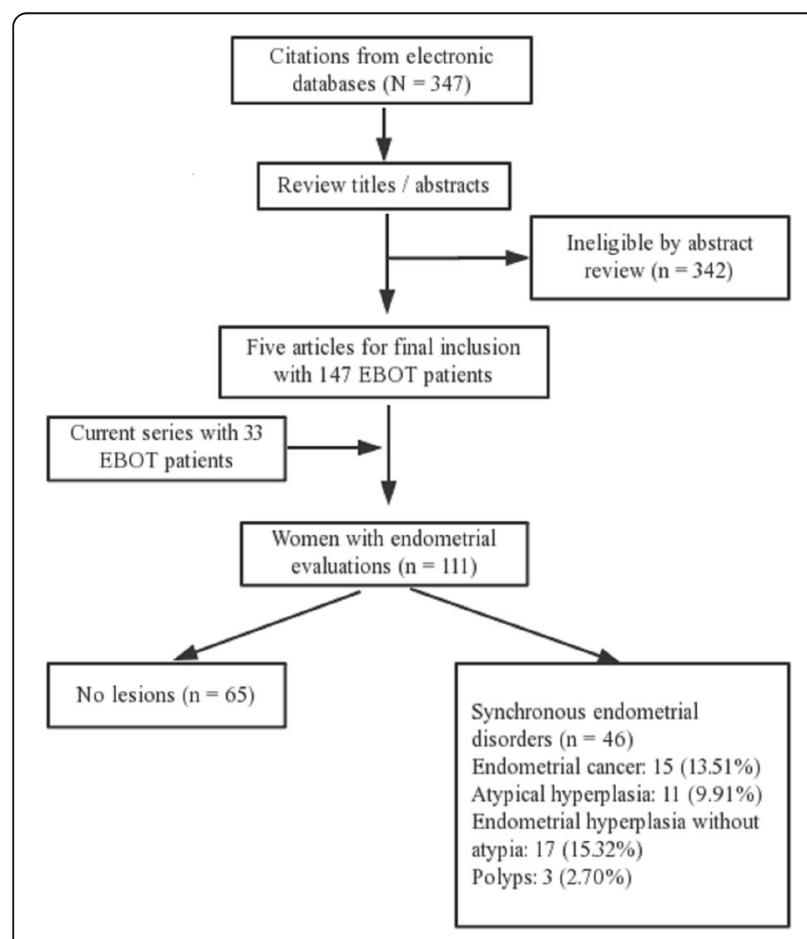

Fig. 1 Literature review of studies of women with EBOT demonstrated that, although women with EBOT have a favorable prognosis, their fertility outcomes are poor.

When treating EBOT patients, physicians must decide whether to include endometrial curettage as a therapeutic approach. Although few studies have evaluated the incidence of endometrial disorders in women with EBOT, studies by Bell and Kurman and Snyder et al. found that $12.5 \%(3 / 24)$ and $68.4 \%$ (13/19), respectively, of EBOT patients also had endometrial disorders [7, 9]. However, our study revealed that the prevalence of synchronous endometrial disorders was 52\%; this incidence remained high (41.4\%) following systematic analysis. Thus, as recommended by Uzan et al., endometrial sampling should be performed in women with EBOT undergoing conservative surgery; in addition, a hysterectomy should be performed in cases requiring radical treatment $[6,12]$.

The mechanisms underlying endometrial disorders in EBOT patients remain unclear. Although one study indicated that stromal luteinizations were more common in EBOT patients with concurrent endometrial disorders, to our knowledge, no other study has confirmed this result [8]. Analyses of our data showed that EBOT patients who were younger, nulliparous, and had AVB were more likely to experience endometrial disorders. To our knowledge, this is the first study to demonstrate this relationship.

Endometrioid carcinomas of the ovary tend to coexist with various forms of endometrial neoplasia, whereas mutations in several genes, including $\beta$-catenin and PTEN, have been demonstrated to occur concurrently with endometrial cancers and endometrioid ovarian cancers [13]. Furthermore, distinct clinical characteristics, including younger age, obesity, premenopausal status, and nulliparity were demonstrated in women with synchronous primary cancers of the endometrium and ovary [14].

The finding that women with AVB were more likely to have concurrent endometrial disorders was not surprising. Endometrial cancer or atypical hyperplasia occurs in $1.31 \%$ of premenopausal women with AVB, and this incidence can increase to $17.3 \%$ in menopausal women $[15,16]$. However, $88.9 \%(n=10 / 11)$ of women with EBOT and AVB also had endometrial disorders, and this incidence was significantly higher than that of the general population. Furthermore, uterine endometrioid adenocarcinomas can also be found in women who do 
not show uterine symptoms [6]. More than $60 \%(8 / 13)$ of our study participants were nulliparous, which is consistent with a study by Eifel et al., wherein $50 \%$ of the study participants who had synchronous endometrioid/ endometrioid tumors were nulliparous [17]. Similarly, Herrinton et al. reported a lower than expected mean parity in women with synchronous endometrial and ovarian cancers [18].

In our study population, women with EBOT and synchronous endometrial disorders were approximately 12 years younger than those with EBOT alone. Similar findings were also reported in women with coexisting endometrial and ovarian carcinomas [19]. The combination of younger age, AVB, and nulliparity, as well as other characteristics, including obesity, in women with synchronous endometrioid/endometrial carcinomas suggests the involvement of a hormonal "field effect" in the development of these simultaneous tumors, particularly endometrioid cell-type tumors [14, 19]. In recent years, accumulating evidence has suggested that ovarian cancer arises from the pleiotropic interactions of the committed stem cells within the ovary, the surrounding microenvironment, and the infiltrating immune cells $[20,21]$. Thus, whether these interactions may contribute to the development of synchronous endometrial and ovarian disorders needs further exploration.

With respect to oncological outcomes, over the past decade several studies have demonstrated the safety of fertility-sparing surgery in early stage epithelial ovarian cancer patients whose preservation of reproductive potential is pivotal to their quality of life [22-24]. Previous studies have found that women with EBOT have a favorable prognosis, with minimal recurrence and no EBOT-associated deaths [6-9]. However, in this study, three patients experienced recurrences, of which two recurred on the contralateral ovary and one relapsed as a bilateral recurrence. All three women were young (23, 30, and 30 years old, respectively), nulliparous, and had synchronous endometrial disorders. Uzan et al. reported a malignant transformation, wherein a 37-year old woman suffered an invasive relapse as endometrioid carcinoma after radical treatment for her first recurrence [6]. This unusual case, in combination with our three recurrences, emphasizes the importance of endometrial biopsy in women with EBOT and the need for an intensive postoperative follow-up, especially in younger patients.

Promising data have been reported for fertility outcomes in women with serous/mucinous BOTs after conservative management. One study reported a pooled estimated spontaneous pregnancy rate of $54 \%$ that increased to $80 \%$ after fertility treatment [4]. In women with advanced-stage serous BOTs, a long-term follow-up study reported a pregnancy rate of 57.1\% [25]. For BOTs, conservative treatment, patient age, and BOT histologic subtype have been reported to influence fertility outcomes; however, for patients with EBOT, there is limited information about fertility outcomes [26-28].

In this study, we demonstrated for the first time that women with EBOT have poor fertility outcomes. Of the nine women who attempted conception, only one (11.1\%) pregnancy was achieved that resulted in a live birth. We should note that five of these nine women developed endometrial disorders after their initial EBOT surgery, including two women with endometrial cancer. Only two women experienced AVB but endometrial sampling was not performed during the initial surgery (unpublished data). Thus, whether nulliparity itself contributes to the development of endometrial disorders or vice versa awaits further study. In addition, adherence and alterations in ovarian function following surgery could also contribute to poor fertility outcomes.

This study has several limitations. First, the present study is inherently limited by its retrospective design and small sample size; in addition, the data were collected from a single institution. As a result, the data in this study did not include all factors that could contribute to the etiology of EBOT tumors, such as body mass index. Women with obesity are at increased risk of endometrial disorders due to excess peripheral conversion of androstenedione to estrone in adipose tissue. However, to the best of our knowledge, due the rare incidence of this condition, this is the largest study to focus on endometrial sampling in women with EBOT. Second, pathological review of the specimens was not performed. The Peking Union Medical College Hospital is a major referral center and has the best gynecology and pathology department in the country. In addition, two gynecological pathologists reviewed original pathology reports. Finally, not all women included in this study underwent endometrial biopsies during their initial surgery (endometrial disorders were observed in six women during follow-up), which may have resulted in overestimation of the incidence of synchronous endometrial disorders. However, this incidence of was $41.4 \%$ following after systematic analysis.

\section{Conclusions}

In summary, the present study demonstrated that endometrial disorders are common in women with EBOT, especially in those who are younger, nulliparous, and have AVB. Thus, endometrial sampling should be performed in women with EBOT undergoing conservative surgery, and a hysterectomy should be performed in cases requiring radical treatment. Although there were no negative oncological outcomes, the fertility rates for these patients were poor, implying that a more active approach should be taken for EBOT patients who wish to become pregnant. 


\section{Abbreviations}

AH: Atypical hyperplasia; AVB: Abnormal vaginal bleeding; BOT: Borderline ovarian tumors; BSO: Bilateral salpingo-oophorectomy; EBOT: Endometrioid borderline ovarian tumors; IEC: Intraepithelial carcinoma

\section{Acknowledgements}

We thank all of the patients for participating in our study.

\section{Funding}

This study was supported by the National Key R\&D Program of China (2017YFC1001200)

\section{Availability of data and materials}

The datasets used and/or analyses performed during the study are available from the corresponding author upon reasonable request.

\section{Authors' contributions}

SJ, JZ, and JL contributed substantially to the study conceptualization, and design, acquisition of data, analysis and interpretation of data, and manuscript writing. JY, YX, and ZL contributed to the interpretation of data and performed critical revisions of important intellectual content. All authors gave approval for the publication of the final version.

\section{Ethics approval and consent to participate}

The Institutional Review Board at the Peking Union Medical College Hospital approved the study (approval No. S-K351). Verbal informed consent was obtained from all patients at their follow-up interviews, and the study was conducted in accordance with the Declaration of Helsinki Principles and regulations of our institute.

\section{Competing interests}

The authors declare that they have no competing interests.

\section{Publisher's Note}

Springer Nature remains neutral with regard to jurisdictional claims in published maps and institutional affiliations.

\section{Author details}

${ }^{1}$ Department of Obstetrics and Gynecology, Peking Union Medical College Hospital, Chinese Academy of Medical Science \& Peking Union Medical College, Beijing 100730, People's Republic of China. ${ }^{2}$ Department of Pathology, Peking Union Medical College Hospital, Chinese Academy of Medical Science \& Peking Union Medical College, Beijing, People's Republic of China.

Received: 11 February 2018 Accepted: 29 March 2018

Published online: 19 April 2018

\section{References}

1. Cadron I, Leunen K, Van Gorp T, Amant F, Neven P, Vergote I. Management of borderline ovarian neoplasms. J Clin Oncol. 2007:25:2928-37.

2. Ellenson LH, Carinelli SG, Cho KR, Kim KR, Kupryjanczyk J, Prat J, et al. Endometrioid tumours. In: Kurman RJ, Carcangiu ML, Herrington CS, Young RH editors. WHO classification of Tumours of female reproductive organs. Lyon: IARC; 2014. p. 29-32

3. Uzan C, Nikpayam M, Ribassin-Majed L, Gouy S, Bendifallah S, Cortez A, et al. Influence of histological subtypes on the risk of an invasive recurrence in a large series of stage I borderline ovarian tumor including 191 conservative treatments. Ann Oncol. 2014:25:1312-9.

4. Darai E, Fauvet R, Uzan C, Gouy S, Duvillard P, Morice P. Fertility and borderline ovarian tumor: a systematic review of conservative management, risk of recurrence and alternative options. Hum Reprod Update. 2013;19: 151-66.

5. Morice P, Uzan C, Fauvet R, Gouy S, Duvillard P, Darai E. Borderline ovarian tumour: pathological diagnostic dilemma and risk factors for invasive or lethal recurrence. Lancet Oncol. 2012:13:e103-15.

6. Uzan C, Berretta R, Rolla M, Gouy S, Fauvet R, Darai E, et al. Management and prognosis of endometrioid borderline tumors of the ovary. Surg Oncol. 2012;21:178-84.
7. Bell KA, Kurman RJ. A clinicopathologic analysis of atypical proliferative (borderline) tumors and well-differentiated endometrioid adenocarcinomas of the ovary. Am J Surg Pathol. 2000;24:1465-79.

8. Bell DA, Scully RE. Atypical and borderline endometrioid adenofibromas of the ovary. A report of 27 cases. Am J Surg Pathol. 1985;9:205-14.

9. Snyder RR, Norris HJ, Tavassoli F. Endometrioid proliferative and low malignant potential tumors of the ovary. A clinicopathologic study of 46 cases. Am J Surg Pathol. 1988;12:661-71.

10. Roth LM, Emerson RE, Ulbright TM. Ovarian endometrioid tumors of low malignant potential: a clinicopathologic study of 30 cases with comparison to well-differentiated endometrioid adenocarcinoma. Am J Surg Pathol. 2003;27:1253-9

11. Prat J. Staging classification for cancer of the ovary, fallopian tube, and peritoneum. Int J Gynecol Obstet. 2014;124:1-5.

12. Harter P, Gershenson D, Lhomme C, Lecuru F, Ledermann J, Provencher DM, et al. Gynecologic Cancer InterGroup (GCIG) consensus review for ovarian tumors of low malignant potential (borderline ovarian tumors). Int J Gynecol Cancer. 2014;24:S5-8.

13. Kurman RJ, Shih I. Molecular pathogenesis and extraovarian origin of epithelial ovarian cancer-shifting the paradigm. Hum Pathol. 2011:42:918-31.

14. Soliman PT, Slomovitz BM, Broaddus RR, Sun CC, Oh JC, Eifel PJ, et al. Synchronous primary cancers of the endometrium and ovary: a single institution review of 84 cases. Gynecol Oncol. 2004;94:456-62.

15. Pennant ME, Mehta R, Moody P, Hackett G, Prentice A, Sharp SJ, et al. Premenopausal abnormal uterine bleeding and risk of endometrial cancer. BJOG. 2017:124:404-11.

16. Ozdemir S, Celik C, Gezginc K, Kiresi D, Esen H. Evaluation of endometrial thickness with transvaginal ultrasonography and histopathology in premenopausal women with abnormal vaginal bleeding. Arch Gynecol Obstet. 2010;282:395-9.

17. Eifel P, Hendrickson M, Ross J, Ballon S, Martinez A, Kempson R. Simultaneous presentation of carcinoma involving the ovary and the uterine corpus. Cancer. 1982:50:163-70.

18. Herrinton LJ, Voigt LF, Weiss NS, Beresford SA, Wingo PA. Risk factors for synchronous primary endometrial and ovarian cancers. Ann Epidemiol. 2001;11:529-33

19. Caldarella A, Crocetti E, Taddei GL, Paci E. Coexisting endometrial and ovarian carcinomas: a retrospective clinicopathological study. Pathol Res Pract. 2008:204:643-8.

20. Giannakeas V, Sopik V, Narod SA. A model for ovarian cancer progression based on inherent resistance. Gynecol Oncol. 2016;142:484-9.

21. Laganà AS, Sofo V, Vitale SG, Triolo O. Epithelial ovarian cancer inherent resistance: may the pleiotropic interaction between reduced immunosurveillance and drug-resistant cells play a key role? Gynecologic Oncology Reports. 2016;18:57-8.

22. Wright JD, Shah M, Mathew L, Burke WM, Culhane J, Goldman N, et al. Fertility preservation in young women with epithelial ovarian cancer. Cancer. 2009:115:4118-26.

23. Kwon YS, Hahn HS, Kim TJ, Lee IH, Lim KT, Lee KH, et al. Fertility preservation in patients with early epithelial ovarian cancer. J Gynecol Oncol. 2009;20:44-7.

24. Vitale SG, Rosa VLL, Rapisarda AMC, Laganà AS. Fertility preservation in women with gynaecologic cancer: the impact on quality of life and psychological well-being. Hum Fertil. 2018;21:35-8.

25. Uzan C, Kane A, Rey A, Gouy S, Duvillard P, Morice P. Outcomes after conservative treatment of advanced-stage serous borderline tumors of the ovary. Ann Oncol. 2010:21:55-60.

26. Palomba S, Falbo A, Del NS, Rocca M, Russo T, Cariati F, et al. Ultra-conservative fertility-sparing strategy for bilateral borderline ovarian tumours: an 11-year follow-up. Hum Reprod. 2010;25:1966-72.

27. Kanat-Pektas M, Ozat M, Gungor T, Dikici T, Yilmaz B, Mollamahmutoglu L. Fertility outcome after conservative surgery for borderline ovarian tumors: a single center experience. Arch Gynecol Obstet. 2011;284:1253-8.

28. Fauvet $R$, Demblocque E, Morice P, Querleu D, Darai E, Behavior of serous borderline ovarian tumors with and without micropapillary patterns: results of a French multicenter study. Ann Surg Oncol. 2012:19:941-7. 